\title{
ACUTE INTERSTITIAL PNEUMONIA (HAMMAN-RICH SYNDROME) - A LIFE THREATENING RESPIRATORY DISEASE
}

\author{
MD. MAHMUDUR RAHMAN SIDDIQUI ${ }^{1}$, MD. MOSTAFIZUR RAHMAN ${ }^{2}$, MAJ. GEN. MD. ABDUL MOYEED \\ SIDDIQUI $^{3}$, HASAN ALI MASUM ${ }^{4}$, RUBAYAT SHEKH GIASUDDIN ${ }^{5}$
}

\begin{abstract}
:
Acute interstitial pneumonia (AIP) is an idiopathic lung disease characterized by rapidly progressive dyspnoea, respiratory failure developing over days to weeks in patients without pre-existing lung disease. AIP show a very poor prognosis, with high mortality and remains unfamiliar to physicians. In this report, we present a case of a mid-age male with AIP who, was treated with intensive medical facilities, still showed a rapid progressive clinical deterioration, and eventually died of respiratory failure.
\end{abstract}

Key words: Acute interstitial pneumonia (AIP), respiratory failure, acute respiratory distress syndrome (ARDS).

Received: 10 May 2017

Accepted: 28 May 2017

\section{Introduction:}

Acute interstitial pneumonia (AIP) is an idiopathic lung disease characterized by rapidly progressive dyspnoea, respiratory failure developing over days to weeks in patients without pre-existing lung disease or extrathoracic disorders known to be associated with lung involvement. ${ }^{1,2}$ AIP is also known as Hamman-Rich syndrome. In 1935, Hamman and Rich first described pulmonary fibrosis of unknown cause in 4 patients with acute diffuse interstitial fibrosis who died within 1-6 months of presentation. ${ }^{3,4,5}$ Acute interstitial pneumonia, which occurs over a wide range of ages, with an approximate mean age of $54 .^{6}$

AIP is radiologically and physiologically resembles acute respiratory distress syndrome (ARDS). ${ }^{5}$ The chest radiographic and high resolution computed tomography (HRCT) scan manifestations of AIP are bilateral and sometimes patchy, and there are alveolar densities associated to areas of ground glass attenuation. ${ }^{5}$ Lung biopsy is confirmatory for AIP and it shows the organizing form of diffuse alveolar damage
(DAD) pattern. ${ }^{7}$ Due to the rarity of this entity, the clinical profile, laboratory data and treatment are not well defined. High-dose corticosteroids and cyclophosphamide are the drugs usually used. 5,8

However, AIP show a very poor prognosis, with high mortality and remains unfamiliar to physicians. In this report, we present a case of a mid-age male with AIP who, was treated with intensive medical facilities, still showed a rapid progressive clinical deterioration, and eventually died of respiratory failure.

\section{Case Report:}

A 60-year-old previously healthy male presented with progressive dyspnea, chest discomfort and productive cough without fever for 10 days. He did not have any other known family history of certain disease. There was no evidence of systemic infection, immune suppression, exposure to toxin, pre-existing lung disease or collagen vascular disease. Initially he was treated in an OPD with antibiotics and bronchodilator and showed good response and advised for follow up

1. Associate Professor of Medicine, Anwer Khan Modern Medical College.

2. Professor of Medicine, Anwer Khan Modern Medical College.

3. Ex-DGMS \& Professor of Medicine, Bangladesh Armed Forces.

4. Registrar, Department of Medicine, Anwer Khan Modern Medical College.

5. Assistant Professor of Medicine, Anwer Khan Modern Medical College.

Address of Correspondence: Dr. Md. Mahmudur Rahman Siddiqui, Associate Professor of Medicine, Anwer Khan Modern Medical College. E-mail: dr.mahmud99@yahoo.com

Bangladesh J Medicine 2017; 28 : 91-94 
visit after 10 days. But he could not report for follow up visit because of some familial circumstances. Unfortunately, he again developed same symptoms after 2 weeks and reported to us.

On physical examination, his body temperature was $99^{\circ} \mathrm{F}$ and blood pressure was $90 / 60 \mathrm{mmHg}$. His respiratory rate was $40 / \mathrm{min}$ and chest wall movement was bilaterally restricted with bilateral crackles in the base of his lungs. Chest x-ray showed bilateral ground glass opacity in middle and lower zone (Fig. 1). High resolution computer tomography (HRCT) of chest showed bilateral ground-glass attenuation with diffuse alveolar consolidation with associated traction bronchiectasis on chest (Fig. 2,3). Because of ongoing hypoxemia and decreased level of consciousness, endotracheal intubation was performed immediately for mechanical ventilation and admitted in intensive care unit (ICU).

Arterial blood gas analysis gave $\mathrm{pH} 7.40, \mathrm{PaO} 288$ $\mathrm{mmHg}, \mathrm{PaCO} 246 \mathrm{mmHg}, \mathrm{SaO} 2$ 80\% (10 L/min of oxygen with face mask), and the white blood cell count was $10,500 / 1 / 4 \mathrm{~L}$ with $76 \%$ neutrophils. C-reactive protein was negative. Serologic examinations including anti-nuclear antibody (ANA), Sm, Jo-1, Scl70, SSA, SSB, P-ANCA, Rheumatoid factor (RF), CANCA, and HIV were all negative. ECG demonstrated sinus tachycardia and other chemistry results were all within normal limits. Blood and sputum culture did not show any abnormal findings. Bronchoscopy was performed and the results suggested absence of microorganism infection or tumor cell and eosinophil count was normal. Cytology and culture results for mycobacterium, fungus, and bacteria were all negative. Bronchoalveolar lavage(BAL) fluid test results for cytomegalovirus, chlamydia, Legionella, herpes simplex virus, and respiratory syncytial virus were negative too. Transbronchial lung biopsy (TBLB) showed slight proliferation of inflammatory and fibrous tissue, diffuse alveolar damage (DAD) associated with hyaline membrane formation, pulmonary interstitial edema, and immature collagen edema, and focal type II pneumocyte hyperplasia were also visible. Based on the clinical,histological and radiologic features she was diagnosed with Acute interstitial pneumonia (AIP).

The patient was treated with high-concentration oxygen therapy, intravenous broad-spectrum antibiotics therapy, antiviral and intravenous application of methylprednisolone in conjunction with invasive ventilation. But all of the treatments were unremarkable. Despite all possible treatments, he died on the 10th day of admission from refractory respiratory failure .

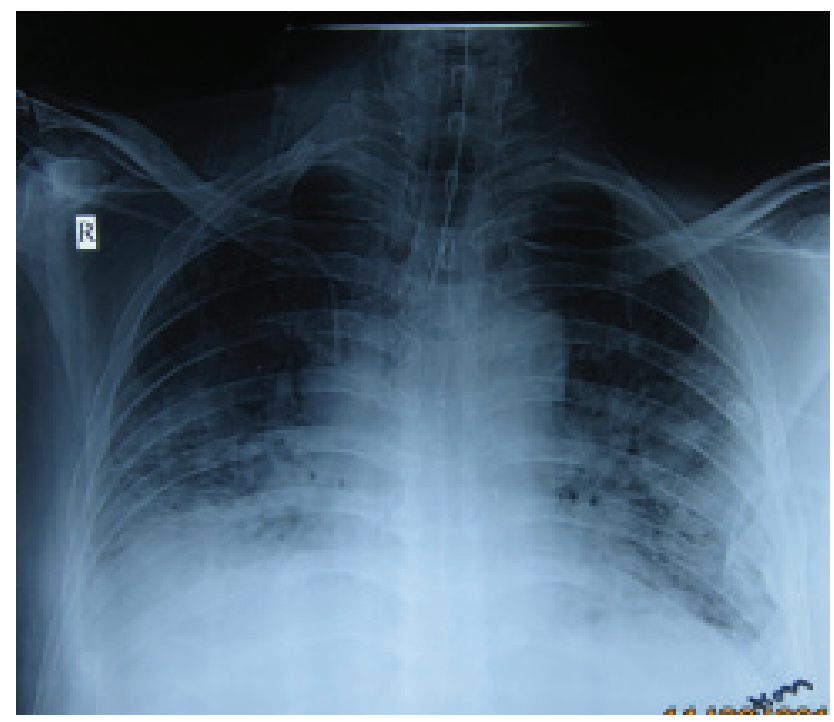

Fig.-1: Chest radiograph with remarkable reduction of lung volume as well as increased lung markings.

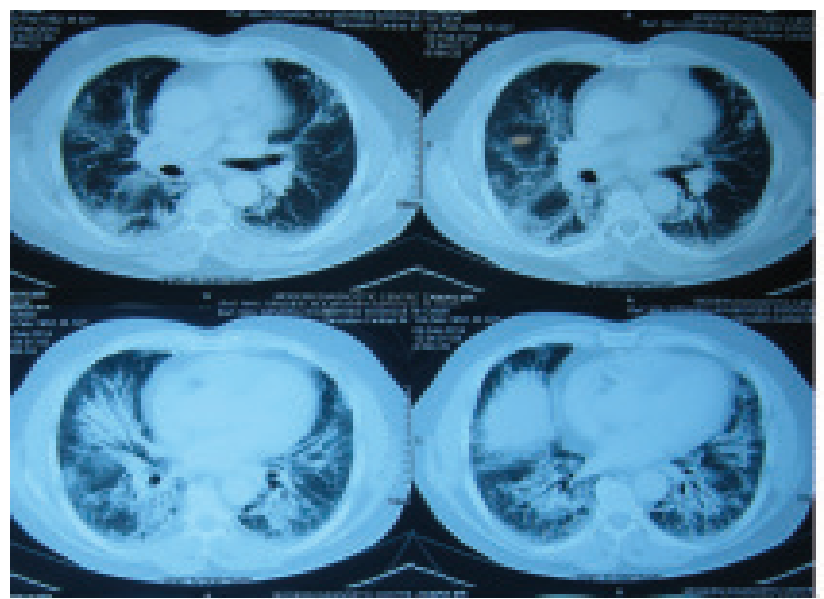

Fig 2: HRCT depicting diffuse areas of pulmonary infiltration, a bilateral geographic distribution of ground glass opacity and consolidation in the more dependent lung with associated traction bronchiectasis.

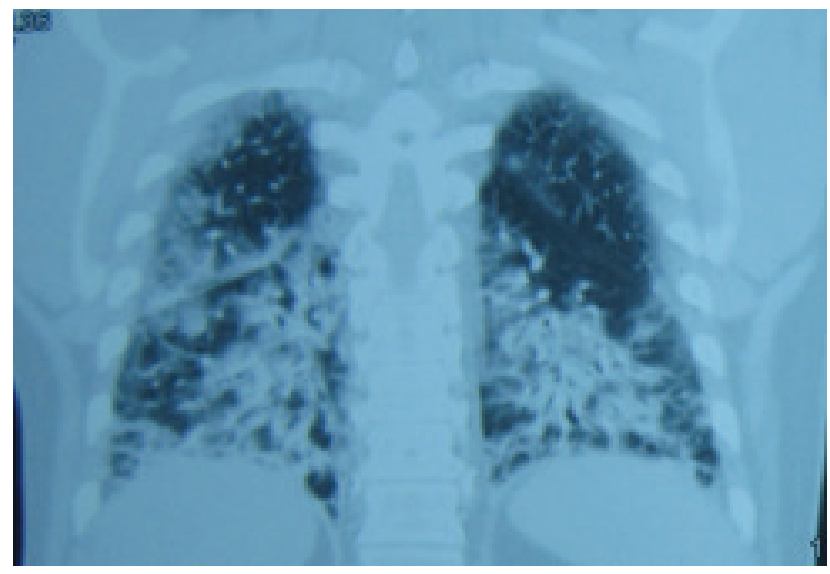

Fig 3: HRCT showing diffuse areas of pulmonary infiltration, a bilateral geographic distribution of ground glass opacity and consolidation with traction bronchiectasis. 


\section{Discussion:}

AIP is a rare disease of lung interstitium, characterized by progressive dyspnea, chest discomfort and cough, with unfavorable prognosis and usually requiring mechanical ventilation. ${ }^{1,2}$ Since many clinical and pathological features of AIP are similar to those of adult respiratory distress syndrome (ARDS), it can be difficult to differentiate. AIP is frequently confused with acute multilobar infectious pneumonia, with other forms of interstitial pneumonia, such as acute exacerbation of idiopathic pulmonary fibrosis, rapidly progressive bronchiolitis obliterans-organising pneumonia or idiopathic acute eosinophilic pneumonia, with collagen-vascular diseases involving the lungs and with primary or secondary pulmonary capillaritis. ${ }^{5}$

Most patients are adults, but AIP has been reported in a wide age range from as young as 7 to over 80 years old, with a mean age of 54 years old. ${ }^{9}$ No gender variation exists. In comparison, the overall US incidence of idiopathic pulmonary fibrosis (IPF) is an estimated 6.8-16.3 per 100,000 person-years, with an increased incidence with advancing age. ${ }^{10}$ The diagnosis of AIP confirm the absence of a specific etiologic agent or event. Most patients describe an upper respiratory tract infection/viral-like prodrome and a nonproductive cough. A bimodal distribution appears to exist, with about $50 \%$ of patients presenting with symptoms of dyspnea within the first week of onset and others presenting after at least a month from the onset of symptoms. ${ }^{9}$ But some patients progress rapidly from shortness of breath to hypoxemic respiratory failure that requires prolonged mechanical ventilation.

Physical examination and laboratory findings are nonspecific. The diagnosis of AIP is very complex because a long list of disorders needs to be excluded. AIP diagnosed by excluding known causes of acute lung injury by history, physical examination, and investigations. The radiographic findings of AIP, overlap with those of ARDS. As in ARDS, the earliest phase begins with a subtle increase in interstitial markings with rapid development of a diffuse alveolar pattern. HRCT scans show bilateral and symmetric, diffuse ground glass and alveolar consolidation opacities. Traction bronchiectasis and bronchiolectasis appear as the disease progresses. Honeycomb fibrosis may be seen in the later phase of the disease. It has been reported that patients with AIP tend to have a more symmetrical and lower zone distribution of abnormalities as well as more honeycombing than patients with ARDS. ${ }^{5,7,11}$
Transbronchial biopsy can be attempted in the early stage of the disease, primarily to exclude other etiologies such as infection. Biopsy findings from patients with AIP may vary on the basis of duration of disease. ${ }^{10,12}$ The initial stages show diffuse alveolar damage, usually in the proliferative or organizing phase, although hyaline membrane remnants can be seen. ${ }^{13}$ The interstitial fibrosis is diffuse, temporally uniform and characterized by extensive fibroblastic and myofibroblastic proliferation with relatively little collagen deposition. The prominence and the uniformity of the fibroblastic/ myofibroblastic proliferation distinguish AIP from the other types of idiopathic interstitial pneumonia. Currently, no specific or proven effective treatments for AIP exist, management is largely supportive, and multidisciplinary team involvement is best for optimal therapy. ${ }^{10}$ Broad-spectrum antibiotics, antiviral agents, high-dose steroids and immunosuppressive therapy are often administered on an empiric basis. ${ }^{5,8,9}$ Mortality in acute case is estimated to $50 \%$ or more. Most deaths occur within 1-3 months of diagnosis. ${ }^{9.10}$

\section{Conclusion:}

Although we could not do much to help in survival of our patient with AIP, the pulmonologists have reached the opinions that the earlier intervention is associated with higher survival rates and better outcome.

Conflict of Interest: We have no conflict of interest.

\section{Acknowledgement:}

Lt. Col. Dr. Shahid, Chief Consultant, Intensive Care Unit, Anwer Khan Modern Medical College Hospital.

\section{References:}

1. Bouros D, Nicholson AC, Polychronopoulos V, du Bois RM. Acute interstitial pneumonia. Eur Respir J 2000; 15: 412-418.

2. Poletti V, Kitaichi M. Facts and controversies in the classification of idiophatic interstitial pneumonia. Sarc Vasc Diff Lung Dis 2000; 17: 229238.

3. Hamman L, Rich AR. Fulminating diffuse interstitial fibrosis of the lungs. Trans Am Clin Climat Assoc 1935; 51: 154-163.

4. Hamman L, Rich AR. Acute diffuse interstitial fibrosis of the lung. Bull Hopkins Hosp 1944; 74: 177-212.

5. Bonaccorsi A, Cancellieri A, M. Chilosi M, et al. Acute interstitial pneumonia: report of a series. Eur Respir J 2003; 21: 187-191. 
BJM Vol. 28 No. 2

6. Bouros D, Nicholson AC, Polychronopoulos V, et al. Acute interstitial pneumonia. European Respiratory Journal 2000; 15(2):412-418.

7. Olson J, Colby TV, Elliot CG. Hamman Rich syndrome revisited. Mayo Clinic Proc 1990; 65: 1538-1548.

8. Schwarz MI. The acute (noninfectious) interstitial lung disease. Compr Ther 1996; 22: 622-630.

9. Vourlekis JS. Acute interstitial pneumonia. Clin Chest Med. 2004 Dec. 25(4):739-47, vii. [Medline]

10. Disayabutr S, Calfee CS, Collard HR, et al. Interstitial lung diseases in the hospitalized patient. BMC Med. 2015 Sep;25(13):245.
Acute Interstitial Pneumonia (Hamman-Rich Syndrome)

11. Tomiyama N, Muller NL, Johkoh T, et al. Acute respiratory distress syndrome and acute interstitial pneumonia: comparison of thin-section CT findings. J Comput Assist Tomogr. 2001 Jan-Feb. 25(1):2833.

12. Tabaj GC, Fernandez CF, Sabbagh E, et al. Histopathology of the idiopathic interstitial pneumonias (IIP): a review. Respirology. 2015 Aug. 20 (6):873-83.

13. Myers JL, Katzenstein AL. Beyond a consensus classification for idiopathic interstitial pneumonias: progress and controversies. Histopathology. 2009 Jan. 54(1):90-103. 